\title{
Histopathological Evaluation of Non-infectious Erythematous Papulo- squamous Skin Lesions in North-West India
}

\author{
Poonam Sharma $^{1 *}$, Ritu Bhagat ${ }^{2}$, Surbhi Mahajan ${ }^{3}$ \\ ${ }^{1}$ Lecturer, Department of Pathology, Government Medical College, Jammu, India \\ ${ }^{2}$ Assistant Professor, Department of Pathology, Government Medical College, Doda, India \\ ${ }^{3}$ Resident, Department of Pathology, Government Medical College, Jammu, India
}

DOI: $\underline{10.36348 / \mathrm{sjpm} .2020 . v 05 i 09.001}$

| Received: 31.08 .2020 | Accepted: 08.09.2020 | Published: 12.09 .2020

*Corresponding author: Poonam Sharma

\section{Abstract}

Background: Papulo-squamous lesions of skin include a heterogeneous group of disorders, often showing overlap in morphology and distribution of lesions, leading to difficulty in diagnosis. Distinct histopathological features and clinical correlation provides a definite diagnosis in these lesions. Aim/Objectives: To evaluate the spectrum of non-infectious erythematous papulo-squamous skin lesions from North West India. Material and Methods: This retrospective diagnostic analytical study was carried out in the Department of Pathology in a tertiary care centre in North-West India. Skin punch biopsies of clinically diagnosed non-infectious, erythematous papulo-squamous skin disorders were included. Diagnosis was confirmed on histopathological examination. Clinical \& histopathological diagnosis of each patient was prepared \& correlation performed. Results: Majority of patients were seen in $4^{\text {th }}$ decade with a male predominance. Lichen Planus was the commonest histopathological lesion (42.9\%) followed by psoriasis (22.9\%). 5 cases of seborrheic dermatitis, 3 cases of pityriasis rosea and 2 cases each of prurigo nodularis and lichen planus pigmentosus were also seen. Clinico-histopathological concordance was observed in $77.1 \%$ cases. Conclusions: Lichen Planus was the commonest papulo-squamous lesion observed in our study. Key histopathological findings and clinico-pathological correlation provides a conclusive diagnosis. Specific histomorphological diagnosis is paramount in distinguishing these lesions as the treatment and prognosis varies widely.

Keywords: Histopathology; Lichen Planus; Psoriasis; Papulo-squamous.

Copyright @ 2020: This is an open-access article distributed under the terms of the Creative Commons Attribution license which permits unrestricted use, distribution, and reproduction in any medium for non-commercial use (NonCommercial, or CC-BY-NC) provided the original author and sources are credited.

\section{INTRODUCTION}

Skin is a complex organ in the human body and skin problems are commonly encountered health problem in India. Prevalence of skin lesions range from $6.3-11.16 \%$ [1]. Skin has a limited number of reaction patterns with which it may respond to pathological stimuli and so clinically different lesions may show similar histological patterns [2]. Interpretation of skin biopsies requires the identification and integration of two distinct morphological features- the tissue reaction pattern and the pattern of inflammation [2].

Papulo-squamous disorders are a heterogeneous group of disorders with unknown etiology. They include a diverse group of inflammatory conditions of the skin characterized by an eruption that exhibit papule and squamous components [3]. Numerous other skin lesions like connective tissue disorders, infections and malignancies may mimic the non-infectious papulo-squamous skin lesions. So histopathological examination is required for confirmation of the diagnosis. Histopathology remains the gold standard for most dermatological diagnosis [4]. So the current study was carried out to analyse the histomorphological features of non infectious papulosquamous lesions of skin with clinico-pathological correlation.

\section{MATERIAL AND METHODS}

This retrospective diagnostic analytical study was conducted for a period of two years in Department of Pathology from January 2017 to December 2018. All patients with non-infectious papulo-squamous disorders, who attended the dermatology outpatient department and underwent skin biopsy (punch/excision) during the study period, were included in the study. Infectious papulo-squamous lesions, lupus erythematosus, dry forms of eczema and chronic dermatitis were excluded from the study. The sociodemographic and clinical data pertaining to patient's age, sex and anatomical site were recorded from the 
requisition forms and data registers. Biopsy specimens received in the department were subjected to routine processing, slides stained with $\mathrm{H} \& \mathrm{E}$ stain and final histopathological diagnosis made. Clinical \& histopathological data of each patient was tabulated \& clinic-histopathological correlation performed.

\section{RESULTS}

The study comprised of cases of skin diseases presenting as papulo-squamous lesions. Majority of cases were in the age group of 31 to 40 years $(37.1 \%)$ followed by 41 to 50 years $(22.9 \%)$ (Table 1$)$. Females were more commonly affected $(57.1 \%)$ compared to males $(42.9 \%)$ with female to male ratio of 1.33 . Majority of the lesions were seen in Limbs (62.85\%) followed by the trunk $(22.85 \%)$ and head, neck and face $(14.30 \%)$.
On histopathological examination, lichen planus was the commonest pathology observed in our study $(42.9 \%)$ followed by psoriasis $(22.9 \%)$ (Table 2$)$. Seborrheic dermatitis was seen in $14.3 \%$ cases while Pityriasis Rosea was seen $8.6 \%$ cases. 2 cases each of Prurigo nodularis and lichen planus pigmentosus were also seen.

Clinico-histological correlation was performed in all cases. A concordance between clinical diagnosis and histopathological diagnosis was observed in $77.1 \%$ cases (Table 3). In lichen planus, clinico-histological agreement was seen $73.3 \%$ cases while in psoriasis, it was observed in $62.5 \%$ cases (Table 3). $100 \%$ concordance was observed in cases of Seborrheic dermatitis and lichen planus pigmentosus while $66.7 \%$ concordance was seen in cases of Pityriasis Rosea.

Table-1: Distribution of Patients According to Age $(n=35)$

\begin{tabular}{|c|c|c|c|}
\hline Age Group (Years) & Number of Males & Number of Females & Percentage \\
\hline $11-20$ & - & 2 & 5.7 \\
\hline $21-30$ & 3 & 4 & 20 \\
\hline $31-40$ & 5 & 8 & 37.1 \\
\hline $41-50$ & 3 & 5 & 22.9 \\
\hline$>50$ & 4 & 1 & 14.3 \\
\hline
\end{tabular}

Table-2: Distribution of Non-Infectious Erythematous Papulo-squamous Lesions (n=35)

\begin{tabular}{|ll|c|c|}
\hline \multicolumn{1}{|c|}{ Disease } & Number & Percentage \\
\hline 1. & Lichen Planus & 15 & 42.9 \\
2. & Psoriasis & 8 & 22.9 \\
3. & Seborrheic dermatitis & 5 & 14.3 \\
4. & Pityriasis Rosea & 3 & 8.6 \\
5. & Prurigo nodularis & 2 & 5.7 \\
6. & Lichen Planus Pigmentosus & 2 & 5.7 \\
\hline \multicolumn{2}{|c|}{ Total } & 35 & 100 \\
\hline
\end{tabular}

Table-3: Clinico-Histological correlation $(n=35)$

\begin{tabular}{|cl|c|c|}
\hline \multirow{2}{*}{ Histopathological Diagnosis } & \multicolumn{2}{|c|}{ Clinical Diagnosis } \\
\cline { 3 - 4 } & Concordant & Discordant \\
\hline 1. & Lichen Planus & $11(73.3 \%)$ & $4(26.7 \%)$ \\
2. & Psoriasis & $5(62.5 \%)$ & $3(37.5 \%)$ \\
3. & Seborrheic dermatitis & $5(100 \%)$ & - \\
4. & Pityriasis Rosea & $2(66.7 \%)$ & $1(33.3 \%)$ \\
5. & Prurigo nodularis & $2(100 \%)$ & - \\
6. & Lichen Planus Pigmentosus & $2(100 \%)$ & - \\
\hline \multicolumn{2}{|c|}{ Total } & $27(73.3 \%)$ & $8(36.3 \%)$ \\
\hline
\end{tabular}

\section{DISCUSSION}

Various pathological stimuli lead to various tissue reaction patterns showing different set of clinical features and having similar histopathological findings [5]. Histopathology remains the gold standard for most dermatological diagnosis [5]. Improved diagnostic specificity may be achieved with a detailed clinicopathological correlation. Skin biopsies can be easily performed under direct visual control and allow precise clinico-histopathological correlation [5].
In our study, skin biopsies of clinically diagnosed papulo-squamous disorders analysed in the department of pathology from January 2017 to December 2018 were included. Majority of cases in our study were seen in 31-40 years group with female preponderance. The results were similar to previous studies published in literature $[6,7]$.

Majority of the lesions in our study were observed in limbs followed by the trunk, head, neck and face. Most of the cases of lichen planus in our study 
occurred in the limbs followed by the trunk and head, neck and face. The results of the study were similar to previous studies $[2,8,9]$ where arms and legs were the most frequently involved. Most of our cases of psoriasis were seen in the limbs, similar to previous study [2].

Lichen Planus was the commonest histopathological diagnosis reported in our study followed by psoriasis. Similar results were obtained by Chandrika VLK et al. [10], Richa G et al. [11], Ukono BA et al. [12] and Chaudhary Raju G et al. [13]. Lichen planus appears as flat topped, violaceous to erythematous lesion [2]. Histopathological findings in majority of cases of Lichen planus included hyperkeratosis, hypergranulosis and vacuolar degeneration of basal cells in epidermis, acanthosis and dermal inflammation, similar to studies by Gupta $\mathrm{R}$ et al. [11], Younas M et al. [14] and Karumbaiah KP et al. [15]. Civatte bodies were observed in 8 cases in our study. Among 15 cases of lichen planus, clinicopathological concordance was observed in $73.3 \%$ cases, similar to previous studies $[12,16]$.

Psoriasis was seen in $22.9 \%$ cases in our study. Common Histopathological findings in psoriasis included Parakeratosis, Hyperkeratosis, Munro microabscesses, suprapapillary thinning and dermal inflammation and vascular changes. In our study, 3 cases with clinical diagnosis of psoriasis showed discordant picture on histopathology. So overall clinicohistopathological concordance in psoriasis was observed in $62.5 \%$ cases, similar to results of Agrawal $\mathrm{S}$ et al. [16] and Richa G et al. [11]. There is considerable overlap in clinical presentation in psoriasis and due to Kobners phenomenon; psoriasis may develop within areas of contact dermatitis [6].

$100 \%$ clinico-pathological concordance was observed in lichen planus pigmentosus and Seborrheic dermatitis. Lichen planus pigmentosus is seen predominantly in the areas exposed to sun and appears as multiple, hyperpigmented patches over the face and extremities [2]. Pigment incontinence was seen in all cases of lichen planus pigmentosus in our study. In Pityriasis Rosea, clinico-histological correlation was obtained in $66.7 \%$ cases.

Overall out of 35 cases, clinico-histological concordance was observed in $77.1 \%$ cases in our study. Similar observations were recorded by Richa G et al. [11] and Younas M et al. [14]. Chandrika VLK et al. [11] and Agrawal S et al. [16] observed 92\% and 58\% clinico-histological concordance in their study respectively.

\section{CONCLUSIONS}

This retrospective study highlights the histopathological spectrum of non-infectious erythematous papulo-squamous skin lesions. Papulosquamous skin lesions show significant overlap in clinical and histopathological features. Some histopathological features are specific and characteristic for particular disease while few disorders may show some overlap. So a combination of clinical observation and histopathological findings is necessary to provide a conclusive diagnosis.

\section{REFERENCES}

1. Gaikwad, S.L., Kumawat, U.D., Sakhare, N.A., D'costa, G.F. (2016). Histopathological Spectrum of Skin Lesions- Experience at Rural Based Hospital. Int J cur Res. Aug; 8(8): 36223-7.

2. Costa, G.D., Bharambe, B.M. (2010). Spectrum of Non-Infectious Erythematous, Papular and Squamous Lesions of the Skin. Indian J Dermatol, 55(3): 225-8.

3. Fox, B.J., Odom, R.B. (1985). Papulosquamous diseases: a review. $J$ Am Acad Dermatol, 12(4):597-624.

4. Reddy, B.R., Krishna, M.N. (2014). Histopathological spectrum of non-infectious erythematous, papulo-squamous lesions. Asian Pac. J Health Sci, 1(4S):28-34.

5. Saritha, C., Unissa, A., Anil, S. (2018). A ClinicoHistopathological Study on Papulosquamous Skin Lesions- Tertiary Care Hospital. Glob. J. Res. Anal, $7(1): 8-11$.

6. Kedarisetti, V., Kumar, V. K., \& Shalini, C. (2020). Study of noninfectious papulosquamous lesions-An experience at a teritiary hospital. Indian Journal of Pathology and Oncology, 7(1), 43-48.

7. Gelfand, J. M., Weinstein, R., Porter, S. B., Neimann, A. L., Berlin, J. A., \& Margolis, D. J. (2005). Prevalence and treatment of psoriasis in the United Kingdom: a population-based study. Archives of dermatology, 141(12), 1537 1541.

8. Sigurgeirsson, B., \& Lindelöf, B. (1991). Lichen planus and malignancy: an epidemiologic study of 2071 patients and a review of the literature. Archives of Dermatology, 127(11), 16841688.

9. VEGA, M. E., WAXTEIN, L., ARENAS, R., HOJYO, M. T., \& DOMINGUEZ- SOTO, L. U. C. I. A. N. O. (1992). Ashy dermatosis and lichen planus pigmentosus: a clinicopathologic study of 31 cases. International journal of dermatology, 31(2), 90-94.

10. Chandrika, V. K., \& Tadepalli, K. (2020). A clinicopathological study of non-infectious papulosquamous lesions of skin. IP Journal of Diagnostic Pathology and Oncology, 5(2), 141-143.

11. Gupta, R., Gupta, K., Swati, S., Kusum, M. (2020). A Clinico-histopathologic Study of Non-infectious Papulosquamous Lesions of Skin at Tertiary Care Hospital. J Med Sci Res. 8(3):532-40. 
12. Ukonu, B. A., Ibekwe, P. U., \& Abimiku, B. A. (2020). Clinicopathological Correlate of Papulosquamous Skin Disorder in a Tertiary Health Care. Journal of Advances in Medicine and Medical Research, 54-65.

13. Chaudhary Raju, G., Chauhan Ankur, P., Makwana Vaishali, R., \& Modi Khushbu, R. (2015). Study of clinico-histopathological correlation of papulosquamous disorders at tertiary care hospital. Sch J App Med Sci, 3(3B), 1154-8.
14. Younas, M., \& ul Haque, A. (2018). Spectrum of Histopathological Features in Non Infectious Erythematous and Papulosquamous Diseases. International Journal of Pathology, 2430.

15. Karumbaiah, K. P., Anjum, A., \& Mallikarjun, M. (2017). A Histopathologic Study of Papulosquamous Lesions of skin. Indian J Pathol: Res Pract, 6(2).

16. Agrawal, S., Mishra, K. B., \& Gupta, C. M. (2018). Histopathological spectrum of non infectious erythematous, papulo-squamous lesions: at a tertiary care institute. Int J Res Med Sci,6(6), 2072-5. 